\title{
Epistemic Modals \\ and \\ Informational Consequence
}

[This is a penultimate draft. Please quote only from the published version. The paper is already available through Online First at http://www.springerlink.com/content/0039-7857]

\begin{abstract}
Recently, Yalcin (2007) put forward a novel account of epistemic modals. It is based on the observation that sentences of the form ' $\phi \&$ Might $\neg \phi$ ' do not embed under 'suppose' and 'if'. Yalcin concludes that such sentences must be contradictory and develops a notion of informational consequence which validates this idea. I will show that informational consequence is inadequate as an account of the logic of epistemic modals: it cannot deal with reasoning from uncertain premises. Finally, I offer an alternative way of explaining the relevant linguistic data.
\end{abstract}

\section{Yalcin's Puzzle}

Consider a sentence such as

(1) It is raining and it might not be raining.

There is something odd about such sentences: they do not seem to be assertable even though they appear to be consistent. The standard explanation would be to assume these sentences to be Moore-paradoxical. However, Yalcin (2007) has given an argument to the effect that these sentences are contradictory after all. His argument is based on the observation that sentences of the form $\phi \& \neg \diamond \phi$ do not embed under 'suppose' and 'if'. The following clauses strike us as odd, too.

(2) Suppose it is raining and it might not be raining.

(3) If it is raining and it might not be raining, then .... 
It seems that (2) and (3) constitute an unsatisfiable demand: we cannot coherently suppose both that it is raining and that it might not be raining. Now, if sentences of the form $\phi \& \diamond \neg \phi$ were consistent, it would be hard to see how this phenomenon could be explained. For, if they were consistent, one would expect that we can suppose them to be true, or so the argument goes. Call this Yalcin's puzzle. Thus, we would arrive at the following constraint on the logic of epistemic modals:

\section{(Epistemic Contradiction)}

$$
\neg \phi \& \diamond \phi \models \perp \text {. }
$$

But of course, one does not want $\diamond \phi$ to imply $\phi$ :

\section{(Nonfactivity) \\ $\diamond \phi \not \models \phi$.}

The principle of epistemic contradiction is incompatible with the principle of nonfactivity if the consequence relation behaves classically. Yalcin's puzzle can therefore be seen as a challenge for a classical consequence relation.

In addition, let me point out that Yalcin's account is designed to satisfy a third principle:

\section{(Łukasiewicz's Principle)$$
\neg \phi \models \neg \diamond \phi .
$$

This is supposed to be motivated by the idea that once we have established $\neg \phi$, we should not take it to be an epistemic possibility any longer that $\phi$ is true. Note that taken in conjunction with nonfactivity, this principle is also incompatible with a classical consequence relation.

Yalcin offers a solution to his puzzle by developing a semantics for epistemic modals together with a non-classical consequence relation. The basic idea of the semantics is to relativize the truth conditions of sentences containing expressions of epistemic modality to an information parameter $s$. Formally, an information parameter is a set of possible worlds. As usual, the truth-values of sentences are relativized to a possible world as well. ${ }^{1}$ The semantic import of an epistemic modal is characterized by the following recursive clause:

\section{(Epistemic Possibility)}

$[\diamond \phi]^{s, w}$ is true iff $\exists w^{\prime} \in s:[\phi]^{s, w^{\prime}}$ is true.

Correspondingly, epistemic necessity is defined as the dual of epistemic possibility:

\footnotetext{
${ }^{1}$ Actually, Yalcin provides in addition for a context parameter, but since it plays no role in his semantics for epistemic modals, I will simplify things by omitting it.
} 


\section{(Epistemic Necessity)}

$[\square \phi]^{s, w}$ is true iff $\forall w^{\prime} \in s:[\phi]^{s, w^{\prime}}$ is true. ${ }^{2}$

Thus, a claim of epistemic possibility is true just in case there is one world in $s$ at which the relevant sentence is true. And a claim of epistemic necessity is true if all worlds in $s$ verify the relevant sentence. This seems plausible.

Now, given the semantics for epistemic modals, the question arises whether it can be supplemented by a consequence relation in such a way that the constraints Yalcin imposes on the logic of epistemic modals are satisfied. Yalcin fulfills this task by designing a consequence relation - informational consequence — which is based on the notion of acceptance.

The notion of acceptance is defined relative to a state of information $s$. The basic idea is that a sentence $\phi$ is accepted in a state of information $s$ just in case that state excludes $\neg \phi$, i.e. $\phi$ is true at all worlds in $s$. More formally, this can be expressed as follows:

\section{(Acceptance)}

A sentence $\phi$ is accepted in a state of information $s$ iff $\forall w \in s:[\phi]^{s, w}$ is true.

Now to Yalcin's definition of informational consequence. It is defined as the preservation of acceptance:

\section{(Informational Consequence)}

A sentence $\phi$ is an informational consequence of a set of sentences $\Gamma$ just in case the following condition holds for every state of information $s:$ if all sentences in $\Gamma$ are accepted in $s$, then $\phi$ is accepted in $s$.

Formally, I will write $\models_{I}$ for informational consequence. Informational consequence thus defined satisfies the constraints imposed by Yalcin on a consequence relation for epistemic modals.

\section{Informational Consequence as Logical Consequence?}

Informational consequence is a non-classical conception of consequence. Since the classical properties of consequence seem to play a vital role in ordinary reasoning, this is in itself a high price to pay. Even if one were to grant this, there are deeper reasons for giving up informational consequence. I will show (a) that it trivializes the logic of epistemic necessity and (b) that informational consequence

\footnotetext{
${ }^{2}$ Yalcin focusses mainly on epistemic possibility. But see Yalcin (2007: 1014) for a brief introduction of epistemic necessity. I will show later on (in Theorem 2.1) that what informational consequence amounts to is perhaps best understood in terms of epistemic necessity.
} 
is an unreliable guide in situations of uncertainty. Finally, I will characterize informational consequence as the preservation of epistemic necessity.

Informational consequence trivializes the logic of epistemic necessity in the sense that

$$
\phi \models_{I} \square \phi \text { and } \square \phi \models_{I} \phi .
$$

We can infer the main part of this from Łukasiewicz's Principle: $\neg \phi \models_{I} \neg \diamond \phi$. By substituting $\neg \phi$ for $\phi, \neg \neg \phi \models_{I} \neg \diamond \neg \phi$. Eliminating the double negation and replacing $\neg \diamond \neg$ by $\square$ then results in $\phi \models_{I} \square \phi$. Of course, informational

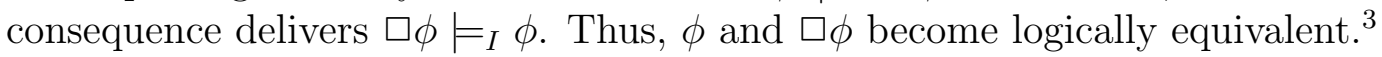

We can see that modal locutions expressing epistemic necessity do not obey a logic which is trivial in the sense above by reflecting on what we are inclined to infer in situations of uncertainty. Compare the following two sentences:

(4) They are at home.

(5) They must be at home.

I might think it quite likely to be true that they are at home because I see that the lights are on. However, I also accept that they might be away because I cannot exclude that they forgot to switch off the lights. Hence, I reject the claim that they must be at home. So, there are situations in which it is rational to have a high credence in (4), but a very low credence (or even credence 0) in (5). But, on Yalcin's account, (5) follows logically from (4). So, we would have to face cases in which it is rational to take a statement to be probable without taking an immediate logical consequence of it to be probable as well.

This clearly violates a reasonable constraint on logical consequence: If a rational and logically omniscient subject's credence function $P$ is such that $P(\phi)=t$, and $\phi=\psi$, then $P(\psi) \geq t$, i.e. if we assign to a statement $\phi$ subjective probability $t$, and we are certain that $\psi$ follows logically from $\phi$, then we should assign to $\psi$ a subjective probability at least as high as $t$. After all, we know that the former cannot hold without the latter. In this sense, informational consequence does not preserve subjective probability. Since this seems to be one of the core features of how logical consequence relates to rational reasoning, we should not accept informational consequence as an account of the logic of epistemic modals. In particular, we should not regard Łukasiewicz's principle as valid.

Once it has been realized that the failure of informational consequence as an account of logical consequence can be identified in situations of uncertainty, we can find further evidence that the principle of epistemic contradiction should be regarded as invalid. By the standard axioms of probability, if two sentences $\phi$ and $\psi$ are contradictory, our credences in $\phi$ and $\psi$ should not sum up to more than 1. More formally, this would give us the following constraint on a fully

\footnotetext{
${ }^{3}$ It should be noted, though, that since the deduction theorem fails for informational consequence, this does not imply that $\phi \equiv \square \phi$ is a theorem in Yalcin's logic.
} 
rational credence function $P$ : if $\phi, \psi \models \perp$, then $P(\phi)+P(\psi) \leq 1$. However, there are situations in which the following two sentences, both being variants of each other, are perfectly acceptable:

(6) Probably they are not at home, but, of course, they might be at home.

(7) Probably they are not at home, but certainly they might be at home.

These sentences are of the form

$$
\text { Probably } \neg \phi \& \text { Of course/Certainly } \diamond \phi .
$$

They express certainty about $\diamond \phi$ and high credence in $\neg \phi$. Thus, they constitute situations in which it seems perfectly acceptable that our credences in $\diamond \phi$ and $\neg \phi$ sum up to more than 1 . Given the argument above, this shows that $\neg \phi$ and $\diamond \phi$ cannot be contradictory.

To confirm, let us replace $\neg \phi$ and $\diamond \phi$ by two sentences which are clearly contradictory.

(8) Probably they are not at home, but, of course, they are at home.

(9) Probably they are not at home, but certainly they are at home.

Indeed, neither of these sentences is acceptable in any epistemic situation. If the present argument is correct, this confirms that the pattern of high credence in $\phi$ and certainty about $\psi$ can only be instantiated by compatible sentences.

For a better diagnosis of what informational consequence amounts to, I will present a theorem to the effect that this consequence relation can be understood as the preservation of epistemic necessity. In what follows, we shall be concerned with a language containing only propositional variables, a complete set of truthfunctional connectives, and a modal operator $\square$ (to be thought of as representing epistemic necessity). To a given set of sentences $\Gamma$, let $\Gamma_{\square}$ be defined as $\{\square \psi \mid \psi \in$ $\Gamma$ \}, i.e. $\Gamma_{\square}$ contains the necessitations of sentences in $\Gamma$. Further, let $\models_{S 5}$ be the standard consequence relation associated with the modal system S5, i.e. $\models_{S 5}$ requires the preservation of truth at every world in every S5-model.

We have the following theorem, a proof of which may be found in the Appendix:

Theorem 2.1. Let $\phi$ and $\Gamma$ be sentences of our language. Then $\Gamma \models_{I} \phi$ just in case $\Gamma_{\square}=_{S 5} \square \phi$.

This theorem says that a sentence $\phi$ is an informational consequence of a set of sentences $\Gamma$ just in case $\square \phi$ is a standard consequence in S5 of the necessitated sentences in $\Gamma^{4}{ }^{4}$

\footnotetext{
${ }^{4}$ Given the terminology of Fitting \& Mendelsohn (1998: 21), it follows from Theorem 2.1 that informational consequence is equivalent to the global consequence relation based on the class of S5-models. Compare the debate concerning the global notion of validity within the framework of supervaluationism. See Varzi (2007) and Williamson (1994: 146f.).
} 
Essentially, Theorem 2.1 shows that informational consequence can be understood as the preservation of epistemic necessity. The conclusion is an informational consequence of the premises just in case the necessitated conclusion is a standard consequence of the necessitated premises. For instance, the principle of epistemic contradiction, $\neg \phi \& \diamond \phi \models_{I} \perp$, holds because $\square \neg \phi \& \square \diamond \phi \models_{S 5} \square \perp$; and $\phi \models_{I} \square \phi$ is valid because $\square \phi=_{S 5} \square \square \phi$.

Now, with respect to metaphysical modality, it would lead to a wrong account of logical consequence if we were to say that a conclusion follows logically from a set of premises just in case it cannot be that the premises are necessarily true without the conclusion being necessarily true. It seems that informational consequence faces a similar problem. An intuitively invalid argument such as $\phi \models$ $\square \phi$ may preserve epistemic necessity: if we are certain about $\phi$, we should perhaps be certain that certainly $\phi$. By focussing only on epistemic situations in which the premises are epistemically necessary, informational consequence validates too many arguments.

\section{An Alternative Explanation}

Yalcin offers informational consequence as the best explanation of a specific kind of linguistic data: the embedding of epistemic modals under 'suppose' and 'if'. Let us recall the sentences of Yalcin's puzzle:

(2) Suppose it is raining and it might not be raining.

(3) If it is raining and it might not be raining, then ....

Clearly, there is something odd about sentences (2) and (3) which needs to be explained. One may offer the proposed inconsistency of the embedded sentence as the best explanation. However, this move is problematic. For there are various sentences containing epistemic modals which do not embed smoothly under 'suppose' and 'if' either, but which are not even remote candidates for being semantically inconsistent. For instance, it is well known that modal adverbs do not embed under 'if'. 5

(10) \# If she is possibly in New York, ...

(11) \# If she is certainly in New York, ...

(12) \# If she is probably in New York, ...

These sentences seem to be defective in more or less the same way as (2) and (3) are. But of course, none of the embedded sentences is semantically inconsistent. ${ }^{6}$

\footnotetext{
${ }^{5}$ Cf. Nilsen (2004).

${ }^{6}$ Note also that it is far from clear that contradictions cannot be embedded under 'suppose' and 'if': there can be a serious dispute about whether a conditional such as 'If she is in New
} 
It is not clear whether modal auxiliaries such as 'might' and 'must' embed smoothly under 'if'. 7 Consider

(13) ?? If she must be in New York, ...

(14) ?? If she might be in New York, ...

Even though not ungrammatical, these sentences seem to have a low degree of acceptability. It may be that the oddness we find with respect to Yalcin's puzzle is nothing but an instance of a more general problem of embedding certain modal locutions.

Quite generally, epistemic modals embed less freely into complex constructions as one would initially expect. For example, disjunctions of sentences flanked by an epistemic modal such as

(15) ?? She might be in New York or she might be in L.A.

are hard to interpret literally. Rather, they tend to be treated as the corresponding conjunction:

(16) She might be in New York and she might be in L.A.

Moreover, wide scope negations of epistemic modals are rare. ${ }^{8}$ On the other hand, epistemic modals embed smoothly into consequents of conditionals, and they live happily in the scope of epistemic attitude verbs such as 'know' or 'believe'. It would be nice to have a general principle which generates the contexts in which epistemic modals are highly acceptable. I have to leave this problem for further research.

Even if one were to ignore the fact that there is a general problem of embedding epistemic modals, there is still an alternative way of explaining the alleged inconsistency of sentences (2) and (3). Yalcin explains it solely in terms of the semantic properties of the embedded sentence 'It is raining and it might not be raining'. Yet one may suspect that the import of 'suppose' and 'if' should be taken into account as well. So, let us see whether one can explain what is odd about sentences like (2) and (3) by drawing on the contribution of 'suppose' and 'if' to such sentences.

York and not in New York, donkeys can speak' is true, as the debate about relevant logic shows; see, for instance, Anderson \& Belnap (1962: 17f.). But if contradictions can be embedded under 'suppose' and 'if', it will not constitute a good explanation to say that certain sentences must be contradictory because they cannot be embedded under these locutions.

${ }^{7}$ I owe this observation to Benjamin Schnieder. See Schnieder (forthcoming) for an expressivist account of epistemic modals based on the limited range of possible embeddings of epistemic modals.

${ }^{8}$ There are also interesting phenomena concerning the interaction of epistemic modals with quantifier phrases. See von Fintel \& Iatridou (2003). 
What is the role of 'suppose'? It seems plausible to characterize it like this: in engaging in a supposition $A$, we temporarily exclude all $\neg A$-possibilities. In the scope of the supposition $A$, we temporarily pretend to act against a background of information which excludes $\neg A$. Following Yalcin, we may model a state of information by a set of worlds $W$. Then the claim would be that in the scope of the supposition $A$ we have $W \subseteq A$, i.e. only $A$-worlds are taken to be possible. Thus, utterances of 'Suppose $A$ ' constitute imperative speech acts, which, if successful, create temporarily a context in which the state of information contains only $A$ worlds.

This proposal allows us already to explain some basic facts. When a part of a conversation is governed by the supposition that she is in New York, it is inappropriate to say 'She is in L.A.' because the possibility that she is in L.A. has been temporarily excluded by supposing that she is in New York. Furthermore, we can observe that within the scope of the supposition that she is in New York, it is even inappropriate to say 'It might be that she is in L.A.' This is because an epistemic modal occurring in the scope of a supposition will be sensitive to the state of information created by the supposition. Given that 'Suppose $A$ ' creates temporarily a state of information $W$ which contains only $A$-worlds, the statement 'Might $\neg A$ ' will be false with respect to $W$. In other words, a supposition $A$ restricts the domain of the epistemic modals occurring in its scope to $A$-worlds only.

On the basis of this description, we may explain why there is something odd about the sentences Yalcin considers. Asking us to suppose that it is raining and it might not be raining may constitute a demand which we cannot fulfill: it requires to exclude temporarily the possibility that it is not raining (by the pragmatic import of 'suppose') and to allow simultaneously for the possibility that it is not raining (by the semantic role of 'might'). Supposing 'It is raining and it might not be raining' would, by the first conjunct, create temporarily a state of information which consists only of worlds in which it is raining. But according to such a state of information, it will not be possible that it is not raining. The supposition that it is raining which would be part of the supposition that it is raining and that it might not be raining restricts the domain of the epistemic modal to rain-worlds. Since epistemic modals within the scope of a supposition are evaluated relative to the state of information created by this supposition, there is no way for 'It might not be raining' to be true as part of the supposition that it is raining.

Thus, the defect in the relevant suppositions would be explicable without invoking a semantic inconsistency in the corresponding sentences. It is sufficient to assume a pragmatic constraint on how suppositions interact with the domain of epistemic modals occurring in their scope. Given that epistemic modals are evaluated relative to the state of information created by the supposition, they do not assume the wide scope interpretation which they would assume outside the scope of the supposition. For this reason, the interpretation of 'It might not be 
raining' which would make 'It is raining and it might not be raining' true is not available in the scope of supposing that it is raining. But supposing that it is raining and that it might not be raining would constitute a context in which 'It might be raining' is true in the scope of supposing that it is raining, since the latter is a part of the whole supposition. ${ }^{9}$

A similar explanation can be developed for sentence (3) if one is prepared to assimilate the indicative 'if' to 'suppose'. ${ }^{10}$ On such a view, the antecedent clause of an indicative conditional invites us to suppose the antecedent to be true. Now, in cases where such a supposition is impossible, one can explain the feeling that the indicative clause does not get off the ground. So, the suggestion would be that the 'if'-clause 'if it is raining and it might not be raining' does not get off the ground because it requires to suppose that it is raining and it might not be raining. But as we have seen above, the air of inconsistency in such suppositions can be explained without invoking a semantic inconsistency of the embedded sentence.

Acknowledgements. Many thanks to Benjamin Schnieder and two anonymous referees for very helpful and stimulating comments.

\footnotetext{
${ }^{9}$ At this stage, a formal treatment of suppositions within speech act theory, which can model relative assertions and the influence of suppositions on the domain of epistemic models, would be welcome. This is a problem for further research.

${ }^{10} \mathrm{I}$ am alluding here to the suppositional view of indicative conditionals. See, for instance, Edgington (1995).
} 


\section{Appendix}

The general strategy for proving the theorem will be to translate Yalcin's proposal into the standard framework of possible worlds semantics.

There is one complication. Yalcin considers sentences with a fixed linguistic meaning. But in order to determine the formal logic his account gives rise to, we should be able to abstract from this feature. The simplest way to do this is to take into account valuations $V$ which assign truth-values to pairs consisting of a propositional variable and a possible world.

The crucial step in translating Yalcin's proposal into the possible worlds framework is the definition of the relevant accessibility relation. ${ }^{11}$ Since Yalcin's clause for epistemic modals is given in terms of the information parameter $s$, we need to tie the accessibility relation to $s$. Recall that $s$ is a set of possible worlds. Now, start with a set of possible worlds $W$ and a subset $s \subseteq W$. For possible worlds $w, w^{\prime} \in W$, let us then define

$$
w R_{s} w^{\prime} \leftrightarrow \text { df. } w^{\prime} \in s
$$

The accessibility relation $R_{s}$ thus defined is quite peculiar: a word $w$ accesses a world $w^{\prime}$ just in case $w^{\prime}$ is in $s$. We shall soon see that this makes Yalcin's clause for the modal operator equivalent to the standard clause for modal operators within the corresponding Kripke model.

Before we can show this, we need a rigorous definition of the class of Kripke models which is apt to characterize Yalcin's semantics. So, let $W$ be a non-empty set of possible worlds, $s$ a non-empty subset of $W$, and $V$ a valuation. ${ }^{12}$ Then we shall call the triple $\left\langle W, R_{s}, V\right\rangle$ a Yalcin model. Hence, a Yalcin model is a certain type of Kripke model delineated by the accessibility relation $R_{s}$. So, the standard definition of truth at a world in a Kripke model carries over to Yalcin models.

I will use the following formalism. A set of sentences $\Gamma$ is said to be valid in a model $M$, formally $M \models \Gamma$, if for all sentences $\phi \in \Gamma: \phi$ is true at all worlds in $M$; in the case of a single sentence $\phi$, I write $M \models \phi$ for $M \models\{\phi\}$. Moreover, I write $M \models_{w} \phi$ if $\phi$ is true in $M$ at world $w$; similarly, let us write $M \models_{w} \Gamma$ if all sentence in $\Gamma$ are true in $M$ at $w$.

What we have to show now is that Yalcin's recursive clause for the modal operator is equivalent to the standard clause of truth in the corresponding Kripke

\footnotetext{
${ }^{11}$ Yalcin's semantics differs from standard possible worlds semantics by treating the state of information as an additional parameter which can be shifted by various operators. This allows for an original take on the interaction between epistemic modals and other epistemic expressions. However, the following will show that once the proposal is restricted to epistemic modals only, it can be modeled within standard possible worlds semantics.

${ }^{12}$ As far as I can see, Yalcin does not require $s$ to be non-empty. But since every sentence is accepted in the empty state of information according to his definition, it is easily seen that requiring $s$ to be non-empty does not alter the definition of informational consequence.
} 
model. To see this, let a set of worlds $W$, a non-empty information parameter $s \subseteq W$, and a sentence $\square \phi$ be given. Further, let $M=\left\langle W, R_{s}, V\right\rangle$ be the Yalcin model defined by these parameters (where we take $V$ to be any fixed valuation). We need to show that, for any world $w \in W, M \models=_{w} \square \phi$ just in case $[\square \phi]^{s, w}$ is true according to Yalcin's definition. So, let $w$ be any world in $W$ and suppose inductively that our claim is true for $\phi$. By applying the standard definition of truth in a Kripke model, $M \models_{w} \square \phi$ iff for all worlds $w^{\prime} \in W$ such that $w R_{s} w^{\prime}$, $M \models{ }_{w^{\prime}} \phi$. By applying the definition of $R_{s}$, the latter is the case iff for all worlds $w^{\prime} \in s, M==_{w^{\prime}} \phi$. By the induction hypothesis, this holds iff for all worlds $w^{\prime} \in s$, $[\phi]^{s, w^{\prime}}$ is true. But this is just Yalcin's clause for the modal operator, i.e. it is the case iff $[\square \phi]^{s, w}$ is true according to Yalcin's definition.

Thus we have shown that Yalcin's semantics can directly be translated into the possible worlds framework by defining an appropriate accessibility relation.

Let us now translate Yalcin's notions of acceptance and informational consequence into the framework of possible worlds semantics. Recall Yalcin's definition of acceptance:

\section{(Acceptance)}

A sentence $\phi$ is accepted in a state of information $s$ iff $\forall w \in s:[\phi]^{s, w}$ is true.

We can define a corresponding notion of acceptance in a Yalcin model:

\section{(Acceptance in a Yalcin model)}

A sentence $\phi$ is accepted in a Yalcin model $\left\langle W, R_{s}, V\right\rangle$ iff

$\forall w \in s: M \models_{w} \phi$.

This has the following consequence:

Proposition 4.1. A sentence $\phi$ is accepted in a Yalcin model $M=\left\langle W, R_{s}, V\right\rangle$ just in case $\phi$ is valid in the Kripke model $\left\langle s, R_{s}, V\right\rangle$.

Proof. Simply note that the worlds in $W$ which are not in $s$ play no role in the definition of acceptance. Also, they do not come into play by the clause for the modal operator: the only worlds which are relevant for evaluating modal sentences are worlds within $s$, too.

Now let us turn to Yalcin's definition of informational consequence:

\section{(Informational Consequence)}

A sentence $\phi$ is an informational consequence of a set of sentences $\Gamma$ just in case the following condition holds for every state of information $s:$ if all sentences in $\Gamma$ are accepted in $s$, then $\phi$ is accepted in $s$.

Accordingly, let us define the relevant counterpart of informational consequence within the possible worlds framework (I will continue to write $\models_{I}$ for the consequence relation thus defined): 


\section{(Informational Consequence*)}

Let $\phi$ be a sentence of our language and $\Gamma$ a set of such sentences. Then $\Gamma \models_{I} \phi$ iff for all Yalcin models $M$ : if all sentences $\Gamma$ are accepted in $M$, then $\phi$ is accepted in $M$.

Given this definition, we can apply Proposition 4.1 to take a first step towards a characterization of informational consequence:

Proposition 4.2. Let $\phi$ be a sentence of our language and $\Gamma$ a set of such sentences. Then $\Gamma \models_{I} \phi$ just in case for all Kripke models $M=\left\langle s, R_{s}, V\right\rangle$ : if $M \models \Gamma$, then $M \models \phi$.

Proof. By definition, $\Gamma \models_{I} \phi$ iff acceptance is preserved in all Yalcin models. Applying Proposition 4.1, a sentence is accepted in a Yalcin model $\left\langle W, R_{s}, V\right\rangle$ just in case it is valid in the corresponding Kripke model $\left\langle s, R_{s}, V\right\rangle$. Hence, $\Gamma \models_{I} \phi$ just in case the argument preserves validity in all Kripke models of the form $\left\langle s, R_{s}, V\right\rangle$.

Before we go on, I need to review some facts and definitions. A Kripke model $\langle W, R, V\rangle$ is an $S 5$-model iff $R$ is an equivalence relation. Further, let us call a Kripke model $\langle W, R, V\rangle$ universal if $R$ is the universal relation on $W$. Obviously, every universal Kripke model is an S5-model (but not every S5-model is universal). Note that in a Kripke model of the form $\left\langle s, R_{s}, V\right\rangle$ the relation $R_{s}$ is, by definition, the universal relation on $s$. Hence, the Kripke models of the form $\left\langle s, R_{s}, V\right\rangle$ are precisely the universal models. Now, it is easily seen that an inference preserves truth at a world in all $S 5$-models iff it preserves truth at a world in all universal models, i.e. S5 is already complete with respect to the class of universal models. ${ }^{13}$ We will make use of this fact in the proof below.

Now to the proof of Theorem 2.1: $\Gamma \models_{I} \phi$ just in case $\Gamma_{\square} \models_{S 5} \square \phi$.

Proof of Theorem 2.1. By Proposition 4.2, $\Gamma \models_{I} \phi$ just in case for all universal models $M$ : if $(M \models \Gamma)$, then $(M \models \phi)$. Start by assuming the latter condition to hold and suppose that the set $\Gamma_{\square}$ is true at a world $w$ in a universal model $M$. Since all sentences in $\Gamma_{\square}$ are boxed, and the accessibility relation is universal, all sentences in $\Gamma$ will be valid in $M$, i.e. $M \models \Gamma$. Therefore, $M \models \phi$ and $\square \phi$ will be true at $w$. Conversely, assume that $\Gamma_{\square} \models_{S 5} \square \phi$. Take any universal model $M$ such that $M \models \Gamma$. By necessitation, $M \models \Gamma_{\square}$. Since $\Gamma_{\square} \models_{S 5} \square \phi$, for all worlds $w$ in $M, M \models \models_{w} \square \phi$, i.e. $M \models \square \phi$. Then $M \models \phi$ by reflexivity. This finishes the proof.

As a corollary, we can infer that the theorems corresponding to informational consequence are precisely the theorems of S5:

Corollary 4.1. Let $\phi$ be any sentence of our language. Then $\models_{I} \phi$ just in case $\models_{S 5} \phi$.

\footnotetext{
${ }^{13}$ For a proof see Priest (2001: 53).
} 
Proof. Let $\phi$ be any sentence of our language. By Theorem 2.1, $\models_{I} \phi$ iff $\models_{S 5} \square \phi$. Hence, what one needs to show is that $\models_{S 5} \square \phi$ just in case $\models_{S 5} \phi$. But this is easy. If $\models_{S 5} \phi$, then, by necessitation, $\models_{S 5} \square \phi$. Conversely, assume $\forall_{S 5} \phi$. Then there is a S5-model $M$ and a world $w$ in $M$ such that $M \nvdash_{w} \phi$. By reflexivity, $M \nvdash_{w} \square \phi$, and therefore $\nvdash_{S 5} \square \phi$. 


\section{References}

[1] Anderson, A.R. \& Belnap, N.D. (1962): "Tautological Entailments", Philosophical Studies 13, 9-24.

[2] Edgington, D. (1995): "On Conditionals", Mind 104, 235-329.

[3] von Fintel, K. \& IAtridou, S. (2003): "Epistemic Containment", Linguistic Inquiry 34, 173-198.

[4] Fitting, M. \& Mendelsohn, R.L. (1998): First-Order Modal Logic, Dordrecht: Kluwer.

[5] Nilsen, Ø. (2004): "Domains for adverbs", Lingua 114, 809-847.

[6] Priest, G. (2001): An Introduction to Non-Classical Logic, Cambridge: Cambridge University Press.

[7] Schnieder, B.: "Expressivism concerning epistemic modals", forthcoming in Philosophical Quarterly.

[8] Varzi, A.C. (2007): "Supervaluationism and Its Logics", Mind 116, 633675.

[9] Williamson, T. (1994): Vagueness, London and New York: Routledge. Page references to the reprint of (2002), London and New York: Routledge.

[10] Yalcin, S. (2007): "Epistemic Modals", Mind 116, 983-1026. 